\title{
Capsular types, LPS genotypes, and virulence-associated genes of Pasteurella multocida strains isolated from pigs")
}

\author{
RUN CHENG LI, CHAO FANG, WEI LUO*, MENG GE, YU LI HU, \\ DUN ZHAO, REN KE QING, XING LONG YU
}

College of Veterinary Medicine, Hunan Agricultural University, Changsha, China

*Huaihua Vocational and Technical College, Huaihua, China

\section{Li R. C., Fang C., Luo W., Ge M., Hu Y. L., Zhao D., Qing R. K., Yu X. L. \\ Capsular types, LPS genotypes, and virulence-associated genes of Pasteurella multocida strains isolated from pigs}

\section{Summary}

Pasteurella multocida (P. multocida), an opportunistic zoonotic pathogen associated with high morbidity and mortality in livestock, shows significant temporal and geographical variation in its serotype distributions and phenotypic characteristics. The aim of this study was To investigate capsular types, lipopolysaccharide (LPS) genotypes, and virulence-associated genes of $P$. multocida strains isolated from pigs. A total of 801 samples (lungs, tonsils, nasal swabs) were collected from slaughterhouses and various regions of the Hunan province. $P$. multocida strains were isolated from various samples, classified, and virulence-associated genes were detected by polymerase chain reaction (PCR). 124 P. multocida strains were assigned to six groups based on both capsular type and LPS genotype, namely A: L3 (capsular type A and LPS genotype 3, 64/124); A: L6 (16/124); D: L6 (38/124); F: L3 (4/124); L3 (1/124) and 1 untypable strain. Of the 23 virulence-associated genes investigated in this study, 14 were highly expressed in $98 \%$ to $100 \%$ of the 124 strains. While tbpA was undetectable in any of the isolated strains, hsf-1, pfhA, tadD, toxA, pmHAS, hgbA, hgbB, and nanB showed differential distribution among the strain groups. Interestingly, pfhA (Mutation or inactivation of pfhA was reported to decrease the virulence of $P$. multocida) was found in $46 \%$ of group A: L3 strains and in $100 \%$ of group F: L3 strains, but not found in other groups. Further investigation is needed to determine whether strains in group A: L3 show greater virulence than the A: L6 P. multocida strains.

Keywords: capsular types, LPS genotypes, $P$. multocida, virulence-associated genes

Pasteurella multocida (P. multocida) is a highly infectious pathogen capable of co-infecting poorly maintained livestock with other pathogens like Mycoplasma hyopneumoniae, Actinobacillus pleuropneumoniae, Bordetella bronchiseptica, porcine reproductive and respiratory syndrome virus, and porcine Circovirus, resulting in severe respiratory diseases or atrophic rhinitis in the swine industry $(4,6,15,17,21,22)$. The serotype distributions and phenotypic characteristics of $P$. multocida show both temporal and geographical variations. In 1952, Carter (5) classified P. multocida into five capsular serotypes (A, B, D, E and F) by indirect haemagglutination test. In 2001, Townsend et al. found that the capsular genotypes of P. multocida strains identified by mPCR were agreeable to Carter's

This work was supported by the National Key Research and Development Program of China (2017YFD0500102). serotyping results. In 1972, Heddleston and Gallagher (14) classified $P$. multocida strains into serotypes 1 through 16 using an agar-gel diffusion precipitin test based on lipopolysaccharide (LPS). LPS of P. multocida lacking the $\mathrm{O}$ antigen, contains a highly conserved inner core and a highly diverse outer core (13). In 2015, Harper et al. reported at least 22 types of outer core in P. multocida, that diversities in LPS were not outlined fully in Heddleston's classification $(12,14)$. Harper et al. designed a rapid LPS-mPCR assay to genotype $P$. multocida strains based on the LPS outer core biosynthesis locus, and classified the strains into eight LPS genotypes: namely LPS genotype L1 covering Heddleston's serotype 1 and 14; L2 covering Heddleston's serotype 2/5; L3 covering Heddleston's serotype 3 and 4; L4 covering Heddleston's serotype 6 and 7; L5 covering Heddleston's serotype 9; L6 
covering Heddleston's serotype 10, 11, 12 and 15; L7 covering Heddleston's serotype 8 and 13; and L8 covering Heddleston's serotype 16 (13).

Capsule and LPS, along with other virulence factors such as adherence proteins ( $p t f A$, fim $A, h s f-1$, $h s f-2, p f h A$ and tadD), toxin (tox $A)$, iron-intake related proteins (exbB, exbD, ton $B, h g b A, h g b B$ and Fur), Sialic acid kinase (nanB and nan $H$ ), hyaluronic acid $(p m H A S)$, superoxide dismutase $(\operatorname{sod} A, \operatorname{sod} C$ and tbpA) and outer membrane proteins (ompA, ompH, oma87 and $p l p B$ ) constitute the virulence factors of $P$. multocida essential for causing acute disease-conditions $(11,18)$. These factors could help P. multocida escape the host immune-surveillance, and help the organism colonize the host-tissue, and elicit an inflammatory response $(11,16)$. Previous studies have reported toxA gene association with $P$. multocida serotype D strains; filament like heamoagglutinin $p f h A$ gene association with $P$. multocida strains of serotypes A, B, E and $\mathrm{F}$; and iron-take associated gene $\operatorname{tbp} A$ association with $P$. multocida strains of serotypes A and B (7).

In this study, tissue and swab samples collected in Hunan (China) between the years 2014 and 2016 were analyzed for $P$. multocida strains. The genotypes of the isolated $P$. multocida strains were characterized by using Townsend's mPCR methods specific for capsule typing (24) and Harper's LPS-mPCR (13). Additionally, 23 virulence-associated genes besides capsule and LPS of $P$. multocida were investigated using PCR methods previously described by Khamesipour et al. (18), and the distribution of these genes among different genotype groups were determined.

\section{Material and methods}

Samples and the reference strain collection. A total of 801 samples were collected between April 2014 and December 2016. Of these, 421 lung and 180 tonsil samples were collected from large slaughter houses in the Hunan province while another 147 lung samples and 53 nasal swabs from pigs with symptoms of fever, cough, and/or dyspnea were submitted from various regions of the Hunan province. The standard types A: 1 (capsular serotype A and LPS serotype 1, LPS genotype L1) P. multocida strain CVCC444 (pmHAS, plpB, tadD were confirmed positive by PCR and sequencing), type B: 2,5 (capsular serotype B and LPS serotype 2,5, LPS genotype L2) P. multocida strain CVCC434, and type D: 3 (LPS genotype L3) P. multocida strain CVCC439 (hgbB, hsf-1, nanB were confirmed positive by PCR and sequencing) were provided by Beijing Bei $\mathrm{Na}$ Chuang Lian Bio-tech Research Institute; the standard types B: 1 (capsular serotype B and LPS serotype 1, LPS genotype L1) P. multocida strain CVCC44-1 were provided by China Institute of Veterinary Drug Control ( $p t f A, f i m A$, hsf-2, pfh $A, \operatorname{exbB}, \operatorname{exbD}, \operatorname{ton} B, h g b A$, Fur, nanH, sodA, sodC, omp $A$, ompH, oma 87 were confirmed positive by PCR and sequencing). The reference strain capsular type $\mathrm{F}$ was isolated and identified by the veterinary laboratory of the Hunan Agricultural University, China (GenBank accession number: KX161714); the reference strain LPS genotype 6 containing toxA gene was isolated and identified by the veterinary laboratory of the Hunan Agricultural University, China (Identification of LPS genotype, GenBank accession number: MH730062. Detection of toxA gene, GenBank accession number: MH730063).

Isolation and culture of $\boldsymbol{P}$. multocida. For tissue samples, small amounts of tonsil or lung tissue (lung from slaughterhouse pigs or sick pigs) were cut from original samples under sterile conditions and weighed before grinding thoroughly. Grinding was performed with a ball mill (MM400Retsch GmbH, Haan, Germany) for 4 min at a frequency of $30 \mathrm{~Hz}$. The homogenates were diluted with $0.9 \%$ normal saline (volume: $10 \times$ tissue sample weight) and $20 \mu 1$ of the diluted homogenates of each sample were inoculated on Blood Agar Base Medium (Barrett Biotechnology (Zhengzhou) Co. LTD, Henan, China), incubated at $37^{\circ} \mathrm{C}$ for $24 \mathrm{hrs}$. Nasal swabs were streaked onto blood agar plates followed by incubation at $37^{\circ} \mathrm{C}$ for $24 \mathrm{hrs}$. All of the inoculated plates were examined for colony formation.

Genomic DNA extraction. Three colonies phenotypically resembling $P$. multocid $a$ were selected from each inoculated blood agar plate and observed by smear microscopy. The colonies with the typical morphologic characteristics of $P$. multocida were enriched and preserved in glycerol and stored at $-80^{\circ} \mathrm{C}$. One representative colony from each tissue sample was picked for genome extraction and used subsequently as a DNA template for PCRs conducted in this study. The genomes from reference strains were simultaneously extracted and used as positive controls. Total DNA of bacterial strains was prepared with Bacteria Genomic DNA Extraction Kit (Takara Biomedical Technology, Beijing, China) following manufacturer's instructions.

Identification of $P$. multocida isolates and determination of capsular type. Primers for genus and capsular classification, described by Townsend (24), were synthesized by Shanghai Bo Shang Biotechnology Co. Ltd. P. multocida-specific primer KMT1 (460 bp) was used to identify $P$. multocida strains from the colonies with the typical morphologic characteristics of $P$. multocida; while primers A (1044 bp), B (760 bp), D (657 bp), E (511 bp) and F (851 bp), specific for each of the types of P. multocida capsule described by Townsend were used in the PCR systems for identification of $P$. multocida strain capsular types. Each PCR reaction sample-mix consisted of $25 \mu 1$ of $2 \times$ Taq Plus Master Mix (TransGen Biotech, Beijing, China), mixed with $22 \mu \mathrm{l}$ of sterile water, $1 \mu \mathrm{l}$ of each of the forward and reverse primers and $1 \mu 1$ of DNA template. The PCR conditions consisted of a 5 min denaturation step at $94^{\circ} \mathrm{C}$, followed by 30 cycles of denaturing at $94^{\circ} \mathrm{C}$ for $30 \mathrm{sec}$, annealing at $55^{\circ} \mathrm{C}$ for $30 \mathrm{sec}$ and elongating at $72^{\circ} \mathrm{C}$ for $45 \mathrm{sec}$, completed with a final extension step at $72^{\circ} \mathrm{C}$ for $7 \mathrm{~min}$. PCR products were analyzed by electrophoresis on $1 \%$ agarose gel.

Identification of LPS genotypes of $\boldsymbol{P}$. multocida strains. The LPS-mPCR method described by Harper et al. (13) was used for LPS genotype identification from genome templates of the selected colonies. Each reaction samplemix contained $25 \mu 1$ of $2 \times$ Taq Plus Master Mix (TransGen Biotech, Beijing, China), mixed with $22 \mu 1$ of sterile water, $1 \mu 1$ of each of the forward and reverse primers and $1 \mu l$ of 
DNA template. The PCR conditions consisted of a $5 \mathrm{~min}$ pre-denaturation step at $94^{\circ} \mathrm{C}$, followed by 30 thermal cycles of denaturing at $94^{\circ} \mathrm{C}$ for $30 \mathrm{sec}$, annealing at $52^{\circ} \mathrm{C}$ for $30 \mathrm{sec}$ and elongation at $72^{\circ} \mathrm{C}$ for $45 \mathrm{sec}$, followed by a final extension step at $72^{\circ} \mathrm{C}$ for $7 \mathrm{~min}$. PCR products were visualized by electrophoresis on $2 \%$ agarose gel.

Detection of virulence-associated genes. 23 virulenceassociated genes were detected by PCR, as described by Khamesipour (18). In brief, each PCR reaction-mix (total volume $=50 \mu \mathrm{l}$ ) consisted of $2 \times$ Taq Plus Master Mix (TransGen Biotech, Beijing, China) $25 \mu$, mixed with sterile water $22 \mu \mathrm{l}, 1 \mu \mathrm{l}$ of each of the forward and reverse primers and DNA template $1 \mu \mathrm{l}$. The PCR and gel electrophoresis conditions were the same as that used for strain determination as described above.

Statistical analysis. Differences in P. multocida positive rates were investigated by chi-squared tests using SPSS software version 19.0 (IBM Inc., Chicago, IL, USA), and the results were considered significant when $\mathrm{P}<0.05$.

\section{Results and discussion}

Isolation of $\boldsymbol{P}$. multocida. Of the 124 P. multocida isolates from 801 samples (described in detail in Table 1), lung samples of slaughterhouse pigs showed higher positive rates $(11.9 \%)$ when compared to those from submitted diseased pigs $(8.2 \%)$. The tonsil tissue samples of slaughterhouse pigs showed the highest positive rates $(23.3 \%)$ compared to any of the other tissue sources, and were significantly higher than lung samples of slaughterhouse pigs $(\mathrm{P}<0.05)$.

Identification of capsular types of $P$. multocida strain. As shown in Table 1, 80 isolates were of capsular type A, 38 of capsular type D, four of $F$ and two untypable by our typing method while capsular types B, $E$ were not found. Of the 50 isolates from slaughtered pig lungs, the positive rate of capsular type A was the highest $(\mathrm{P}<0.05)$ with 49 isolates of capsular type $A$ and one of F. For strains from tonsils of slaughtered pigs, 23 strains were of capsular type A, 27 of D and three of capsular type F. There were no significant differences between the positive rates of capsular type A and of $\mathrm{D}(\mathrm{P}>0.05)$, but the positive rates of $\mathrm{A}$ and $\mathrm{D}$ were significantly higher than other capsular serotypes $(\mathrm{P}<0.05)$. For strains recovered from the submitted lungs, seven were capsular type A strains, four capsular type D strains and one untypable. For strains from submitted nasal swabs, one strain was of capsular type $\mathrm{A}$, seven of $\mathrm{D}$, and one strain was untypable.

Identification of $\boldsymbol{P}$. multocida LPS genotypes. LPS genotyping results showed that 124 strains could be of either LPS genotype L3 or L6 (69 and 54 strains respectively), while one strain was untypable (Tab. 1). For isolates recovered from lungs of slaughtered pigs, 40 strains were of LPS genotype L3 and 10 of L6, and 24 strains recovered from slaughtered pig tonsils were of LPS genotype L3 and 29 of L6. For submitted lungs, five strains were of LPS genotype L3 and seven of L6. For isolates from submitted nasal swabs, eight strains belong to LPS genotype L6 and one strain untypable by the LPS-mPCR.

Classification of $P$. multocida strains based on capsular serotype and LPS genotype. Based on capsular type and LPS genotype, 124 strains were classified into six groups, namely A: L3 (capsular type A and LPS genotype L3, 64/124), A: L6 (capsular type A and LPS genotype L6, 16/124), D: L6 (capsular type D and LPS genotype L6, 38/124), F: L3 (capsular type F and LPS genotype L3, 4/124), genotype L3 (untypable capsular type and LPS genotype L3, 1/124), and the type with untypable capsular and LPS (untypable capsular type and untypable LPS genotype, 1/124) (described in Table 1).

Distribution of virulence-associated genes among different genotypes of $\boldsymbol{P}$. multocida strains. On an average, 17 virulence-associated genes were expressed by each strain. The 49 strains observed in this study carrying the highest number of virulence-associated genes (18 virulence-associated genes), were classified into type A: L3 and A: L6, with 33 and 16 strains respectively. The one untypable by both capsular and LPS PCR systems carried the least number (14) of virulence-associated genes. As shown in Table 2, the average number of $P$. multocida virulence-associated genes was 17 or more in addition to capsular type D. The number (1) of unclassified strains was too small to be calculated.

Fourteen virulence-associated genes (adherence proteins: $p t f A$, fim $A, h s f-2$; iron-intake related proteins: $\operatorname{exbB}, \operatorname{exbD}$, tonB, and Fur; Sialic acid kinase: $n a n H$; superoxide dismutase: $\operatorname{sod} A$, sodC; outer membrane proteins: ompA, ompH, oma87, and plpB) were de-

Tab. 1. Isolation and identification of $P$. multocida from 801 samples

\begin{tabular}{|c|c|c|c|c|c|c|c|c|c|c|c|c|c|}
\hline \multirow{3}{*}{ Sample type } & \multirow{3}{*}{$\begin{array}{c}\text { No. of } \\
\text { samples } \\
\text { examined }\end{array}$} & \multirow{3}{*}{$\begin{array}{l}\text { Positive } \\
\text { rate of } \\
\text { samples }\end{array}$} & \multirow{3}{*}{$\begin{array}{l}\text { No. of } \\
\text { strains }\end{array}$} & \multirow{3}{*}{$\begin{array}{c}\text { No. of } \\
\text { unclassified } \\
\text { strains }\end{array}$} & \multicolumn{9}{|c|}{ Classification method } \\
\hline & & & & & \multicolumn{3}{|c|}{ No. of capsular type } & \multicolumn{2}{|c|}{ No. of LPS type } & \multicolumn{4}{|c|}{ No. of capsular type combined LPS type } \\
\hline & & & & & A & D & $\mathbf{F}$ & L3 & L6 & A: L3 & A: L6 & D: L6 & F: L3 \\
\hline Lungs (slaughtered) & 421 & $11.9 \%$ & 50 & 0 & 49 & 0 & 1 & 40 & 10 & 39 & 10 & 0 & 1 \\
\hline Tonsil (slaughtered) & 180 & $23.3 \%$ & 53 & 0 & 23 & 27 & 3 & 24 & 29 & 21 & 2 & 27 & 3 \\
\hline Lungs (submission) & 147 & $8.2 \%$ & 12 & $1^{\mathrm{a}}$ & 7 & 4 & 0 & 5 & 7 & 4 & 3 & 4 & 0 \\
\hline Nasal swab (submission) & 53 & $17.0 \%$ & 9 & $1^{b}$ & 1 & 7 & 0 & 0 & 8 & 0 & 1 & 7 & 0 \\
\hline Total & 801 & $15.5 \%$ & 124 & 2 & 80 & 38 & 4 & 69 & 54 & 64 & 16 & 38 & 4 \\
\hline
\end{tabular}

Explanations: $\mathrm{a}$ - unclassified strains by capsular typing; $\mathrm{b}$ - unclassified strains by both capsular and LPS typing 
tected in $98 \%$ to $100 \%$ of all 124 recovered strains. The distribution of $h s f-1, p f h A, \operatorname{tad} D$, toxA, pmHAS, hgbA, $h g b B$, and $n a n B$ among strains with different genotypes are described in Table 3. $t b p A$ was not found in this study. $h s f-1$ was found in all D: L6 and F: L3 groups, but was detected in only one strain of capsular type A; $h s f-1$ was more frequently found in LPS genotype L6 strains than in L3. pfh $A$ was found in $29.8 \%$ of the 124 strains, $50 \%$ of type A: L3 strains and $100 \%$ of type $\mathrm{F}$ : L3 were positive for $p f h A$, but it was absent in capsular type D and LPS genotype L6 strains. $t a d D$ was found in $83.8 \%$ of capsular type A strains, with more prevalence in A: L6 strains than A: L3. tadD was not found in capsular types D or $\mathrm{F}$, while toxA was detected only in two capsular type D strains, accounting for $1.6 \%(2 / 124)$. pmHAS was detected in $100 \%$ of capsular type A strains, but not in capsular types D or F. $h g b A$ was found exclusively in type A: L3 (51.6\%). $h g b B$ was found in all strains of type D: L6 and F: L3, but only $48.4 \%$ in type A: L3. nanB was found in all type A: L6 strains and only in another A: L3 (1.6\%) type, accounting for $14.5 \%$ of the total 124 recovered strains.

P. multocida is a zoonotic pathogen that infects both domesticated and wild animals as well as humans with variable disease outcomes based on host species straintype. Classification of P. multocida strains based on serotyping of capsular and LPS types is time-consuming, serum limited. On the other hand, the PCR typing system established by Townsend for capsular genotype of $P$. multocida strains showed results comparable to the capsular serotyping method, and the LPS-mPCR developed by Harper could classify more strains compared to the LPS serotyping method. In this study, using Townsend and Harpers' strategies, we classified the 124 isolates into six groups based on a combination of capsular typing and LPS genotyping. We believe that our classification method is a more elaborate strategy to facilitate the epidemiological study of P. multocida.

As shown in Table 1, the characteristics for capsular type A P. multocida strain isolated in the present study was in accordance with that obtained in previous studies, with percent of type A strain higher than that of other types $(2,10,19)$. Furthermore, The recovered isolates showed differences in both capsular type and LPS genotype prevalence, based not only on tissue type but the source of the swine donor. For example, capsular type D strain was identified in all tissue-sources of submitted diseased swine tissue but not in slaughtered swine lungs. Generally, slaughtered pigs were healthy adults ( $>110 \mathrm{~kg}$ body-weight) while submitted swine weighed $<50 \mathrm{~kg}$. Further investigation is necessary to determine whether: 1) adult pigs are resistant to capsular type D strains rendering the strain unable to colonize the adult lung but only tonsils and nasal passages; or, 2) the lungs could only be infected by type $\mathrm{D}$ if the resistance declines.

Detection of $P$. multocida virulence-associated genes could provide useful data for pathogenesis study, and some of the virulence related antigens could be candidates for developing effective vaccines against P. multocida $(1,3)$. Virulence-associated genes were highly expressed in P. multocida strains analyzed in this study, which agrees well with previous reports $(1,7,9$, 18). However, contrary to previous reports $(7,9)$, we found $h g b A$ to be expressed in $51.6 \%$ of $P$. multocida A: L3 strains, and nanB in $100 \%$ of $P$. multocida A: L6 strains, but no expression in $P$. multocida groups D: L6 and F: L3. The detection rate for $h g b A$ and $n a n B$ in the recovered strains were $27.4 \%$ and $14.5 \%$, respectively, lower than that $(>80 \%)$ reported by researchers in 
Germany (7) and Brazil (9), implying a geographical variation in the two genes in $P$. multocida strains.

tox $A, \operatorname{tbp} A$ and $p f h A$ were regarded as the important epidemiological marker genes for characterizing $P$. multocida field strains $(1,7)$. toxA exists in capsular serotype $\mathrm{D}$ strains and is associated with atrophic rhinitis $(7,20)$. tbp $A$, controlling the receptor for iron binding, which is associated with bovine hemorrhagic septicemia, usually exists in some of the clinical isolates from bovine and sheep $(1,7) . p f h A$ encodes the filament like heamoagglutinin, mutation of $p f h A$ was reported to decrease the virulence of $P$. multocida to mouse, and inactivation of fhaB2 resulted in a high degree of attenuation when turkeys were challenged intranasal $(8,23)$. Among the 124 P. multocida isolates, toxA was detected exclusively in D: L6 P. multocida strains, accounting for $1.6 \%(2 / 124)$. In this study, $p f h A$ exists only in P. multocida A: L3 and type F: L3 strains, with detection rate $46 \%$ and $100 \%$, respectively. Further investigation is necessary to answer whether the virulence of $P$. multocida type A: L3 strain is stronger than A: L6.

\section{References}

1. Aski H. S., Tabatabaei M.: Occurrence of virulence-associated genes in Pasteurella multocida isolates obtained from different hosts. Microb. Pathogenesis 2016, 96, 52-57.

2. Bethe A., Wieler L. H., Selbitz H. J., Ewers C.: Genetic diversity of porcine Pasteurella multocida strains from the respiratory tract of healthy and diseased swine. Vet. Microbiol. 2009, 139, 97-105.

3. Bosch M., Garrido M. E., Rozas A. M. P. D., Badiola I., Barbé J., Liagostera $M$.: Pasteurella multocida contains multiple immunogenic haeminand haemoglobin-binding proteins. Vet. Microbiol. 2004, 99, 103-112.

4. Božidar S., Oliver R., Dubravka J., Ksenija N., Snežana I., Ognjen S., Đorđe C. Dragan K.: Survey of infectious agents associated with Porcine Respiratory Disease Complex (PRDC) in Serbian swine herds using Polymerase Chain Reaction (PCR) detection. Acta. Vet. 2015, 65, 79-88.

5. Carter G. R.: The type specific capsular antigen of Pasteurella multocida. Can J. Med. Sci. 1952, 30, 48.

6. Cowart R. P., Bäckström L., Brim T. A.: Pasteurella multocida and Bordetella bronchiseptica in atrophic rhinitis and pneumonia in swine. Can. J. Vet. Res. 1989, 53, 295-300.

7.Ewers C., Lübke-Becker A., Bethe A., Kiebling S., Filter M., Wieler L. H. Virulence genotype of Pasteurella multocida strains isolated from different hosts with various disease status. Vet. Microbiol. 2006, 114, 304-317.

8. Fuller T. E., Kennedy M. J., Lowery D. E.: Identification of Pasteurella multocida virulence genes in a septicemic mouse model using signature-tagged mutagenesis. Micro. Pathog. 2000, 29, 25-38

9. Furian T. Q., Borges K. A., Laviniki V., Rocha S. L. D. S., Almeida C. N. D. Nascimento V. P. D., Salle C. T. P., Moraes H. L. D. S.: Virulence genes and antimicrobial resistance of Pasteurella multocida isolated from poultry and swine. Braz. J. Microbiol. 2016, 47, 210-216.

10. García N., Fernández-Garayzábal J. F., Goyache J., Dominguez L., Vela A. I.: Associations between biovar and virulence factor genes in Pasteurella multocida isolates from pigs in Spain. Vet. Rec. 2011, 169, 362.

11. Harper M., Boyce J. D., Adler B.: The key surface components of Pasteurella multocida: capsule and lipopolysaccharide. Curr. Top. Microbiol. 2012, 361, 39-51.

12. Harper M., John M., Edmunds M., Wright A., Ford M., Turni C., Blackall P. J., Cox A., Adler B., Boyce J. D.: Protective efficacy afforded by live Pasteurella multocida vaccines in chickens is independent of lipopolysaccharide outer core structure. Vaccine 2016, 34, 1696-1703.

13. Harper M., John M., Turni C., Edmunds M., Michael F. S., Adler B., Blackall P. J., Cox A. D., Boyce J. D.: Development of a rapid multiplex PCR assay to genotype Pasteurella multocida strains by use of the lipopolysaccharide outer core biosynthesis locus. J. Clin. Microbio. 2015, 53, 477-485.

14. Heddleston K. L., Gallagher J. E., Rebers P. A.: Fowl cholera: gel Diffusion precipitin test for serotyping Pasteurella multocida from avian species. Avian. Dis. 1972, 16, 925-936.
15. Holko I., Urbanova J., Holkova T., Kmet V.: Diagnostics of main bacterial agents of porcine respiratory diseases complex (PRDC) using PCR detection of Mycoplasma hyopneumoniae. Vet. Med. Czech. 2004, 49, 35-41.

16. Hunt M. L., Adler B., Townsend K. M.: The molecular biology of Pasteurella multocida. Vet. Microbiol. 2000, 72, 3-25.

17. Kang M. S., Kang M. W., Jung S. H., Lee H. S.: Study on porcine respiratory disease complex from slaughtered pigs in Namwon, Korea. Surf. Coat. Tech. 2013, 112, 67-70.

18. Khamesipour F., Momtaz H., Azhdary M. A.: Occurrence of virulence factors and antimicrobial resistance in Pasteurella multocida strains isolated from slaughter cattle in Iran. Front. Microbiol. 2014, 5, 536.

19. Ki-Eun L., Hye-Young J., Ji-Youn L., Myoung-Heon L., Hwan-Won C., KyungSoo C., Young-Hee O., Dong-Jun A.: Phenotypic characterization and random amplified polymorphic DNA(RAPD) analysis of Pasteurella multocida isolated from Korean pigs. J. Vet. Med. Sci. 2012, 74, 567-573.

20. Lax A. J., Chanter N.: Cloning of the toxin gene from Pasteurella multocida and its role in atrophic rhinitis. J. Gen. Microbiol. 1990, 136, 81-87.

21. Rutter J. M., Taylor R. J., Crighton W. G., Robertson I. B., Benson J. A.: Epidemiological study of Pasteurella multocida and Bordetella bronchiseptica in atrophic rhinitis. Vet. Rec. 1984, 115, 615-619.

22. Takeuti K. L., Watanabe T. T. N., Castro L. A. D., Driemeier D., Barcellos D. E. S. N. D.: Histopathological and immunohistochemical characterization of pneumonia caused by co-infection between Pasteurella multocida and Mycoplasma hyopneumoniae in swine. Acta. Sci. Vet. 2013, 41, 1-6.

23. Tatum F. M., Yersin A. G., Briggs R. E.: Construction and virulence of a Pasteurella multocida fha B2 mutant in turkeys. Microb. Pathog. 2005, 39, 9-17.

24. Townsend K. M., Boyce J. D., Chung J. Y., Frost A. J., Adler B.: Genetic organization of Pasteurella multocida cap Loci and development of a multiplex capsular PCR typing system. J. Clin. Microbiol. 2001, 39, 924-929.

Corresponding author: Prof. Dr. Xing Long Yu, College of Veterinary Medicine, Hunan Agricultural University, Changsha, China; e-mail: xlyu999@126.com 